Aufsatz ÖR

Hannah Birkenkötter und Maximilian Steinbeis

\title{
Rechtswissenschaftliche Blogs in Deutschland - zu Möglichkeiten und Grenzen eines neuen Formats in den Rechtswissenschaften
}

DOI 10.1515/jura-2015-0003

In den letzten zehn Jahren hat sich die juristische PublikatiMonographien und Konferenzen mit anschließenden Tagungsbänden sind nicht länger die einzigen Mittel, juristische Expertise öffentlich zu machen und zur Diskussion zu stellen. An ihre Seite ist ein Medium getreten, das noch vor einem Jahrzehnt einer Welt anzugehören schien, die mit Wissenschaft denkbar wenig zu tun hat. Blogs, einst als unseriöses Gossip-Medium und Domäne radikalisierter Soziopath_innen verschrien, haben sich im recht(swissenschaft)lichen Diskurs als Mittel des Informationsaustauschs und der Debatte fest etabliert.

Im Folgenden wollen wir einen Überblick über die aktuelle recht(swissenschaft)liche Bloglandschaft in Deutschland geben. In einem ersten Schritt wollen wir versuchen, die Entwicklung der juristischen Bloglandschaft in Deutschland nachzuzeichnen, und dabei auf die besondere Rolle anwaltlicher Blogs eingehen. In einem zweiten Schritt werden wir auf der Grundlage der genannten Blogs einige Merkmale festhalten, die Blogs im Unterschied zu anderen Publikationsmöglichkeiten kennzeichnen. Zuletzt wollen wir die Perspektiven aufzeigen, die Blogs für die Rechtskommunikation im Allgemeinen und die rechtswissenschaftliche Kommunikation im Besonderen eröffnen. Das beinhaltet notwendig die Frage nach dem Selbstverständnis der Rechtswissenschaften, die hier - aus einer formatspezifischen Perspektive, die nach dem Wert spezieller Formate für die Rechtswissenschaft fragt - nur angerissen werden kann. onslandschaft in Deutschland gewandelt. Fachzeitschriften,

\section{Als Expertin sichtbar werden: Anwaltsblogs}

Im internationalen Vergleich ist Deutschland mit der Etablierung rechtswissenschaftlicher Blogs eher spät dran. Als sich vor ca. zehn Jahren in den USA die heute noch dominierenden akademischen Blog-Netzwerke bildeten (z.B. das konservativ-libertäre Netzwerk Volokh Conspiracy ${ }^{1}$, sein linksliberales Gegenstück Balkinization ${ }^{2}$ oder die Völkerrechts-Plattform Opinio Juris ${ }^{3}$ ), war es in Deutschland ein Anwalt, der als Pionier die Möglichkeiten des juristischen Bloggens erschloss. Dem Düsseldorfer Strafverteidiger Udo Vetter gelingt es seit 2003, sich mit seinem Lawblog $^{4}$ - einer Mischung aus heiteren Begebenheiten aus dem bunten Leben eines Feld-, Wald- und Wiesenanwalts und tagesaktuellen rechtspolitischen Einwürfen zum Internetrecht - ein in Zehntausenden zu messendes Publikum und ein enormes Renommee als Medienrechtsexperte aufzubauen.

Für Rechtsanwält_innen ist ein Blog ein kaum zu übertreffendes Marketinginstrument. Sie leben davon, mit ihrer Expertise von potenziellen Mandant_innen, die rechtlichen Rat und Informationen suchen, gefunden zu werden. Erfolgreiche Blogposts, die Neuigkeitswert haben oder eine kontroverse These zur Diskussion stellen, werden oft über Facebook, Twitter und andere soziale Medien lawinenartig weiterverbreitet. Zudem werden sie gern von anderen Blogs verlinkt - und das führt dazu, dass sie bei den Suchergebnissen von Google und anderen Suchmaschinen ganz oben auftauchen. Wer im Internet nach Informationen $\mathrm{zu}$ bestimmten Schlagwörtern sucht, wird mit hoher Wahrscheinlichkeit auf diesen Blogpost stoßen.

\footnotetext{
Hannah Birkenkötter: Die Autorin ist wissenschaftliche Mitarbeiterin am Forschungsprojekt »Verfassungsblog: Perspektiven der Wissenschaftskommunikation in der Rechtswissenschaft" sowie am Lehrstuhl für Öffentliches Recht, insb. Verfassungsrecht, und Rechtsphilosophie an der Humboldt-Universität zu Berlin. Maximilian Steinbeis: Der Autor ist Rechtsanwalt und Journalist sowie Gründer und Herausgeber des Verfassungsblogs.
}

1 http://www.volokh.com, heute auf der Website der Zeitung Washington Post zu finden.

2 http://balkin.blogspot.de/.

$3 \mathrm{http://opiniojuris.org.}$

$4 \mathrm{http}: / /$ lawblog.de. 
Von dort aus ist es nur noch ein kleiner Schritt zur Mandatierung. Blogs ermöglichen so zu minimalen Kosten und mit minimalen Streuverlusten und maximaler Effizienz das Ziel jeder anwaltlichen Marketingkommunikation zu erreichen: als Expert_in wahrgenommen und sichtbar zu werden ${ }^{5}$.

Viele erfolgreiche Anwaltsblogs bewegen sich in Rechtsgebieten, die Resonanz im Internet wahrscheinlich machen - Internet- und Social-Media-Recht, Medien- und Urheberrecht, Datenschutz ${ }^{6}$. Doch auch in Rechtsgebieten weitab der öffentlichen Aufmerksamkeit scheint es attraktiv zu sein, einen Blog zu etablieren: So präsentiert sich beispielsweise der Berliner Rechtsanwalt Andreas Jede auf seinem Blog bundesweit als Experte im deutschen Waffenrecht ${ }^{7}$. Die Münsteraner Kanzlei von Kai Behrens schärft mit ihrem Blog ihr Profil als Ansprechpartner für alle Fragen rund um das Handelsvertreterrecht ${ }^{8}$. Nicht jedes exotische Rechtsgebiet liefert indessen genügend Stoff, um hinreichend regelmäßige Blogposts zu generieren. So findet man auf vielen spezialisierten Anwaltsblogs überwiegend kleine Nutzwerttexte ohne aktuellen Anlass, die mangels Neuigkeitswert und/oder kontroversen Thesen kaum verlinkt und weiterverbreitet werden und damit die Sichtbarkeit im Anwaltsmarkt nur geringfügig erhöhen.

Die Notwendigkeit eines scharfen inhaltlichen Profils dürfte auch erklären, warum bislang so wenig Großkanzleien von dieser Marketingmöglichkeit Gebrauch machen. Ihre Expertise ist breit gestreut. Manche in Großkanzleien tätige Anwälte wie der Jones-Day-Partner Johannes Zöttl mit seinem Kartellblog ${ }^{9}$ betreiben ihre Blogs ganz in Eigenregie und tragen ihre Kanzleizugehörigkeit dabei gar nicht nach außen. Eine Ausnahme bildet die Wirtschaftskanzlei CMS Hasche Sigle, die seit 2010 das Blogformat nutzt, um über Neuigkeiten, vor allem höchstrichterliche Rechsprechung, in allen möglichen Feldern des Wirtschaftsrechts zu berichten ${ }^{10}$. Ein Sonderfall ist die Kanzlei Becker Büttner Held, die sich - obzwar mit über 250 Berufsträger_innen eine Großkanzlei - stark auf das Energierecht spezialisiert hat und seit 2011 per Blog ihre aktuelle und potenzielle

5 So belegen Anwaltsblogs aktuell den 3. und 4. Platz des Rankings der Seite »Jurablogs« (http://www.jurablogs.com/blogs/ranking; Stand: 15. August 2014). Das Ranking setzt sich aus einer Kombination von Posts pro Monat und Klickzahlen auf jeden Post zusammen.

6 So z. B. der Blog Internet-Law des Freisinger Anwalts Thomas Stadler (www.internet-law.de) oder der Blog Kriegs-Recht des Berliner Anwalts Henning Krieg (www.kriegs-recht.de).

$7 \mathrm{http} / / /$ deutscheswaffenrecht.de.

8 http://www.handelsvertreter-blog.de/.

$9 \mathrm{http://kartellblog.de.}$

$10 \mathrm{http}: / /$ www.cmshs-bloggt.de/.
Mandantschaft über alle relevanten Entwicklungen im Bereich der Energiepolitik informiert ${ }^{11}$.

\section{Blogs in der deutschen Rechtswissenschaft}

Auch die rechtswissenschaftlichen Fachverlage haben Blogs als Möglichkeit erkannt, sich und der von ihnen vertriebene Expertise mehr Sichtbarkeit und Resonanz zu verschaffen. Auf dem Beck-Blog des Verlags C.H.Beck schreiben 19 Anwälte, Richter, Staatsanwälte und Wissenschaftler (ausschließlich Männer) über ihre jeweiligen Fachgebiete $^{12}$. Der Verlag Wolters Kluwer bietet dem pensionierten Richter und Rechtsblog-Veteran ${ }^{13}$ Detlef Burhoff ein Forum für seinen Blog zu Strafrechts- und Justizthemen $^{14}$.

Aus der Rechtswissenschaft selbst heraus blieben Blogs lange Zeit die Ausnahme. Attraktiv ist das Format offenbar schon länger für Wissenschaftler_innen, die nach dem Ende ihrer Universitätskarriere unbehelligt von den Mühen des akademischen Publikationswesens nach Möglichkeiten suchen, die Öffentlichkeit an ihren Gedanken teilhaben zu lassen. Ein Kreis von Rechtssoziolog_innen um den Berliner Emeritus Dieter Simon verfasst seit 2008 auf dem Mops-Block in bittersüßem Tonfall und ohne Furcht, Anstoß zu erregen, Tagebucheinträge und Lektürenotizen zu Rechtstheorie und Wissenschaftsbetrieb ${ }^{15} . \mathrm{Zu}$ ähnlichen Zwecken, wenngleich weniger polemisch, nutzt Klaus F. Röhl, Emeritus aus Bochum und ebenfalls Rechtssoziologe, seit 2007 seinen RSozBlog ${ }^{16}$. Beide Blogs sind sehr persönlich geprägt, aber offenbar nicht dazu intendiert, unmittelbar kontroverse Resonanz auszulösen. Der RSozBlog lässt zwar Kommentare zu, hat aber nur selten welche (auch Links sind sehr selten, dafür gibt es Fußnoten). Beim Mops-Block sind Kommentare gar nicht erst vorgesehen.

$11 \mathrm{http}: / /$ www.derenergieblog.de (Maximilian Steinbeis berät BBH beim Betreiben des Energieblogs).

12 http://blog.beck.de.

13 Burhoff hatte bis 2010 als "Richter Ballmann" sehr erfolgreich über das Innenleben der Justiz gebloggt, und zwar anonym. Als nach einem Streit Udo Vetters »Lawblog« Burhoffs Anonymität aufhob, stellte dieser seinen Blog vorläufig ein.

$14 \mathrm{http}: / /$ blog.strafrecht.jurion.de.

15 http://www.mops-block.de. Simons Mit-Blogger sind derzeit die emeritierte Frankfurter Rechtshistorikerin Regina Ogorek sowie sein in Rostock forschender Schüler Benjamin Lahusen.

$16 \mathrm{http}: / /$ www.rsozblog.de. 
Deutlich ehrgeiziger sind neuerdings die Versuche aus dem akademischen Nachwuchs, Blogs als Stätten rechtswissenschaftlicher Kommunikation zu etablieren. Seit Januar 2013 veröffentlicht der aus den Assistententagungen für Öffentliches Recht hervorgegangene JuWiss-Blog von Studierenden, Promovierenden und Habilitierenden verfasste Analysen $\mathrm{zu}$ aktuellen verfassungs- und verwaltungsrechtlichen Themen ${ }^{17}$. Der Arbeitskreis junger Völkerrechtswissenschaftler_innen (AjV) veranstaltet seit Mai 2014 auf dem Völkerrechtsblog anspruchsvolle Debatten $\mathrm{zu}$ aktuellen Entwicklungen im internationalen Recht ${ }^{18}$. Betrieben wird der Völkerrechtsblog von Mitgliedern des wissenschaftlichen Nachwuchses, der Autor_innenkreis schließt aber - anders als der JuWiss-Blog - Professor_innen ebenso ein wie Promovierende und Habilitierende oder Mitglieder der völkerrechtlichen Praxis.

Seit 2009 versucht schließlich der Verfassungsblog grenzüberschreitende rechtswissenschaftliche Diskurse zu organisieren und sie so einem größeren Publikum zugänglich zu machen ${ }^{19}$. Seine Liste von über 200 Autor_innen umfasst Studierende und Promovierende genauso wie weltweit anerkannte Professorinnen und Professoren unterschiedlicher Fachrichtungen. Sein Themenspektrum umfasst verfassungsrechtliche Entwicklungen nicht nur in Deutschland, sondern auf europäischer und globaler Ebene und im europäischen und außereuropäischen Ausland.

Damit unterscheidet sich die deutsche rechtswissenschaftliche Blogosphäre deutlich von der anderer Länder, insbesondere den USA: jenseits des Atlantiks waren es vor allem Projekte von einzelnen Wissenschaftlern - neben den bereits genannten Blogs von Eugene Volokh und Jack Balkin auch der Blog "Instapundit « von Glenn Reynold ${ }^{20}$ die die rechtswissenschaftliche Blogszene vorangetrieben haben. Es ist keine Seltenheit, dass amerikanische Professor_innen einen eigenen Blog betreiben ${ }^{21}$. Auch Gruppenblogs, wie $\mathrm{zB}$ der völkerrechtliche Blog Opinio Juris oder der recht neue Blog Just Security ${ }^{22}$ leben maßgeblich von ihren Betreibern: Kevin Jon Heller wird den Leser_innen von Opinio Juris ebenso ein Begriff sein wie Steve Vladeck dem Publikum von Just Security. Auch die französi-

\section{$17 \mathrm{http}: / /$ www.juwiss.de.}

$18 \mathrm{http://voelkerrechtsblog.com.}$

$19 \mathrm{http}: / /$ www.verfassungsblog.de.

$20 \mathrm{http}: / /$ pjmedia.com/instapundit/.

21 Neben Balkinization und Volokh Conspiracy gibt es zahlreiche Einzelblogs, wie etwa http://althouse.blogspot.de, http://ericposner. com, http://dianemarieamann.com. Die »Blog Directory» der American Bar Association listet rund 300 Blogs mit professoraler Autor_innenschaft: http://www.abajournal.com/blawgs/topic/law+pro fessor/ (Stand: 15. August 2014).

22 http://justsecurity.org. sche rechtswissenschaftliche Blogosphäre hat mit Einzelprojekten begonnen: im öffentlichen Recht hatte es etwa der Professor Frédéric Rolin ${ }^{23}$ zu einigem Ruhm gebracht $^{24}$. Solche Einzelprojekte sucht man - mit Ausnahme der beiden schon genannten Emerita-Projekte RSozBlog und Mops-Block - in der deutschen Rechtswissenschaft vergeblich.

\section{Ein Blog: Was ist das überhaupt?}

Warum finden sich in der deutschen Rechtswissenschaft vergleichsweise wenige Versuche, die eigenen Ideen oder Erkenntnisse im Wege des Blogs zu verbreiten? Die Frage danach hängt eng damit zusammen, welches Potenzial man dem Blog als Medium rechtswissenschaftlicher Kommunikation zumisst. Dazu soll im Folgenden zunächst herausgearbeitet werden, was den Blog von anderen Formaten unterscheidet, um dann in einem abschließenden Schritt kritisch nach dem Potenzial des Blogs für die rechtswissenschaftliche Kommunikation zu fragen.

Das vielleicht hervorstechendste Merkmal des Blogformats - gerade im Gegensatz zu den bislang vorherrschenden schriftlichen Formaten wie Fachzeitschriften oder Buchpublikationen - ist der vollständige Wegfall nahezu sämtlicher Publikationshürden. Bloggen kann buchstäblich jede_r. Die Installation erfordert kaum technische Kenntnisse. Die Software ist frei verfügbar, und für das Webdesign gibt es zahllose vorgefertigte und ebenfalls kostenlose Lösungen. Die einzigen Fixkosten, die man aufbringen muss, um einen Blog zu betreiben, ist die eigene Zeit. Im Vergleich zu herkömmlichen Publikationsformen erlauben Blogs obendrein eine Veröffentlichung nahezu in Echtzeit: Zwischen der Niederschrift und Publikation eines Gedankens steht buchstäblich nur ein Mausklick.

Dies dürfte gleichzeitig auch ein Hauptkritikpunkt an diesem Format sein: Da könnte ja jede_r kommen! In der Tat, mit dem Wegfall aller Intermediäre entfällt auch deren

$23 \mathrm{http}$ ://frederic-rolin.blogspirit.com. Weitere Beispiele für erfolgreiche Einzelblogs französischer Rechtswissenschaftler_innen sind etwa der Blog »Liberté, Libertés chéries« der Verfassungsrechtsprofessorin Rosaline Letteron (http://libertescheries.blogspot.com) oder der Blog des Wirtschaftsrechtsprofessors Bruno Dondero (http://bru nodondero.wordpress.com), beide von der Universität Paris I-Sorbonne.

24 Siehe hierzu auch das Interview mit dem französischen Blogbetreiber Serge Slama (http://combatsdroitshomme.blog.lemonde.fr): Slama/Birkenkötter VerfBlog (2014) http://www.verfassungsblog.de/ blogbeitraege-und-andere-formen-des-wissenschaftlichen-arbeitensergaenzen-sich-wunderbar-interview-mit-serge-slama-betreiber-desblogs-combats-pour-les-droits-de/. 
Filterfunktion, und mit der Echtzeitpublikation möglicherweise die nüchterne Selbstkritik, die oft erst in zeitlicher Distanz zum eigenen Text entsteht ${ }^{25}$.

Dies ist das zweite Hauptmerkmal des Blogs im Unterschied zu herkömmlichen Publikationsformaten: Die Filterfunktion wird auf den Zeitraum nach der Publikation verlagert. Wer Unsinn schreibt, verliert Leser_innen. Dazu kommt: wer fehlerhaft informiert oder unhaltbare Thesen verbreitet, wird im Regelfall schonungslos und umgehend korrigiert. Blogs lassen im Unterschied zu Zeitschriftenbeiträgen und anderen gedruckten Formaten zu, dass Leser_innen das Gelesene kommentieren und diese Kommentare weiteren Leser_innen zugänglich machen. In manchem Fall entspinnt sich daraus ein regelrechtes Gespräch, in dem der Inhalt des Blogposts weiter entwickelt werden kann.

Weitere Merkmale sind für einzelne Blogs nur schwer bestimmbar: während sich die Autor_innenhinweise des Verfassungsblogs, JuWiss-Blogs und des Völkerrechtsblogs hinsichtlich der Längenvorgabe (rund 1000 Wörter) und der Forderung nach einer starken These, einem klaren Argument ähneln, sieht die Blogrealität anders aus. Manche Beiträge gehen klar über die Empfehlung der 1000 Wörter hinaus, andere Beiträge sind kürzer. Auch die »Thesenhaftigkeit« der einzelnen Beiträge variiert stark und ist ganz offenkundig von den einzelnen Autor_innen abhängig. Auch wenn es anders als in einer wissenschaftlichen Publikation im herkömmlichen Sinne in einem Blogpost erlaubt und sogar üblich ist, in der ersten Person zu schreiben, machen bei weitem nicht alle Blogger_innen davon Gebrauch. Ob ein Blog besonderen Wert auf Lesbarkeit legt, welche Art von Texten - kurze, niedergeschriebene Gedanken, ausgearbeitete Argumente, Interviews - veröffentlicht werden, all das hängt von den Betreiber_innen und den Autor_innen der jeweiligen Blogs ab. Das eine etablierte Blogformat gibt es nicht. Der Blog ist vor allem ein offenes Format: als relativ neues Phänomen haben sich keine festen Konventionen entwickelt. Das macht den Blog zu einem Experimentierfeld. Oder in den Worten von Blog-Veteran Jack Balkin: "Your persona, and what you want the blog to cover, shapes what people

25 Darauf weist auch Slama in seinem Interview mit dem Verfassungsblog hin: "Scheinbar erlaubt der Blog einem, Positionen ohne Filter (mit Ausnahme vielleicht einer gewissen Selbstzensur) darzustellen, die möglicherweise in einer Zeitschrift schwieriger zu vertreten wären.«, Slama/Birkenkötter VerfBlog (2014) http://www.ver fassungsblog.de/blogbeitraege-und-andere-formen-des-wissenschaft lichen-arbeitens-ergaenzen-sich-wunderbar-interview-mit-serge-sla ma-betreiber-des-blogs-combats-pour-les-droits-de/. Ähnlich auch D’Aspremont EJIL:Talk! (2011) http://ejiltalk.org/in-defense-of-the-ha zardous-tool-of-legal-blogging/. expect of the blog, and gives the blog its general direction $\ll^{26}$.

\section{Perspektiven}

Damit ist aber nicht gesagt, dass das Medium Blog vollständig abgekoppelt wäre von seinem Inhalt. Vielmehr begünstigt das Format des Blogs bestimmte Arten von Texten und damit auch bestimmte Inhalte. Dass potenziell auch »klassische« wissenschaftliche Abhandlungen rein technisch Gegenstand eines Blogposts sein können, kann davon nicht ablenken: ein Zeitschriftenaufsatz auf einem Blog würde dennoch als Zeitschriftenaufsatz wahrgenommen, unabhängig davon, ob er physisch auf bedrucktem Papier oder online bereitgestellt wird ${ }^{27}$. Das Medium Blog begünstigt, wenn man sich die Mehrzahl der bislang erschienenen Blogposts in der deutschsprachigen rechtswissenschaftlichen Blogosphäre anschaut, kürzere, oft von einem konkreten Ereignis angeleitete Texte, die dann gegenseitig in Bezug genommen werden können ${ }^{28}$. Es ist vor allem das Potenzial des Blogs, gesprächsartige Austausche zwischen den einzelnen Autor_innen und ihrer Leserschaft zu ermöglichen, dem für die Rechtswissenschaften eine belebende Wirkung zukommen kann.

Diese These erfordert Erläuterung. Denn sie kann auch missverstanden werden als eine unkritische Präferenz des Gesprächs, der (im Blog höchstens nachgebildeten und daher allenfalls quasi-)mündlichen Kommunikation gegenüber dem geschriebenen Text, die mit ihrem Fokus auf $»$ Lesbarkeit $\aleph^{29}$, auf die Verständigung zwischen Autor_in und Leser_innen hinter die Erkenntnisse des sog. linguistic turn ${ }^{30}$ in den Geisteswissenschaften zurück-

26 Balkin/Birkenkötter/Steinbeis VerfBlog (2014) http://www.verfas sungsblog.de/en/blogging-way-changing-relations-authority-publicsphere-interview-professor-jack-balkin-founder-balkinization-2/.

27 So erscheint etwa das German Law Journal ausschließlich online und nicht in gedruckter Fassung, dennoch stellt niemand in Frage, dass es sich dabei um eine Zeitschrift im traditionellen Sinne handelt: http://www.germanlawjournal.com/index.php?pageID=1.

28 Auf dem Völkerrechtsblog ist dieses Potenzial zum Prinzip erhoben: unter den bislang erschienenen, überwiegend inhaltlich geprägten Texten finden sich jeweils ein "Beitrag « und eine »Replik«, die unmittelbar auf den Ausgangsbeitrag Bezug nimmt. Es wird bereits in den Autor_innenhinweisen auf das zentrale Format »Beitrag Replik« hingewiesen: http://voelkerrechtsblog.files.wordpress.com/ 2014/07/bloghinweise.pdf.

29 Kemmerer VerfBlog (2012) http://www.verfassungsblog.de/ rechts-wissenschaft-sui-generis/.

30 »The linguistic turn « ist ein von dem Philosophen Richard Rorty 1967 herausgegebener Sammelband, der zum Ziel hat »to provide materials for reflection on the most recent philosophical revolution, 
fällt ${ }^{31}$. Ino Augsberg sieht hierin ein logozentristisches Verständnismodell, das die Möglichkeit eines einheitlichen, »richtigen« Verstanden-Werdens als gegeben voraussetze und so ganz traditionell den gerade für die deutsche juristische Methodologie charakteristischen »Unifizierungswunsch« verfestige, eine Tendenz, die noch dadurch verstärkt würde, dass Blogs - weil ein Merkmal immer auch Schnelligkeit ist - vornehmlich an Rechtsprechung anknüpften ${ }^{32}$.

Wir halten diesen Vorwurf für unzutreffend. Die These, Blogs nähmen implizit für sich in Anspruch, gegenüber klassisch-schriftlichen Kommunikationsformen einen Gewinn an Eigentlichkeit herzustellen oder gar die "Autor_in « wieder in ihren Stand als Herrscher_in über ihren Text einzusetzen, hält genauerer Betrachtung nicht stand. Die Präferenz von Blogs für einen umgangssprachlicheren, »mündlicheren« Stil lässt sich auf andere Weise als durch einen solchen Anspruch erklären, nämlich als Ausfüllen technisch neu entstandener Artikulations- und Diskursspielräume.

Oben war bereits von den beiden Hauptmerkmalen des Bloggens die Rede: dem Bedeutungsverlust der Intermediäre - kein Verlag, keine Redaktion, keine Druckerei, kein Versand, nicht einmal mehr Tinte und Papier sind notwendig, damit das Geschriebene und seine Leser_innen einander finden können - sowie der Möglichkeit, dass Lesende das Gelesene in unmittelbarer zeitlicher und räumlicher Nähe zu demselben kommentieren und so ihre Kommentare zum Teil des Textes werden lassen $\mathrm{zu}$ können ${ }^{33}$.

that of »linguistic philosophy«...[i.e.] the view that philosophical problems are problems which may be solved (or dissolved) either by reforming language, or by understanding more about the language we presently use«, Rorty (1967), 3. Damit beschreibt Rorty die Hinwendung der Philosophie zu einer vertieften Auseinandersetzung mit den Voraussetzungen und Funktionsweisen der Sprache, die maßgeblich durch Ludwig Wittgensteins »Philosophische Untersuchungen« (1953) beeinflusst wurde. Wittgenstein legte in den »Philosophischen Untersuchungen « einen Schwerpunkt auf den Gebrauch der Sprache, nachdem er in seinem früheren Werk »Tractatus logico-philosophicus « für eine Lösung philosophischer Probleme durch eine Idealsprache plädiert hatte. In den »Philosophischen Untersuchungen« lehnte er die Möglichkeit einer Idealsprache jedoch ab. Der Begriff »linguistic turn« oder "sprachphilosophische Wende« wird mittlerweile auch allgemein für diese Entwicklung in der Philosophie gebraucht.

31 So Ino Augsberg »Blogozentrismus, Oder: Who's Afraid of Writing and Text?, erscheint in: Funke/Lachmayer (Hrsg.) Formate der Rechtswissenschaft (2015). Das Manuskript liegt den Autor_innen vor.

32 Augsberg ebd.

33 Ein spezielles Phänomen dabei ist, dass Kommentare oft anonym oder pseudonym verfasst werden, was gelegentlich zu destruktivem Verhalten ermutigt.
Die »Mündlichkeit« von Blogposts ist vor diesem technisch bedingten - Hintergrund leicht zu erklären: Bloggen heißt, sich und seine Gedanken zur Diskussion zu stellen. Wer bloggt, präsentiert im Regelfall keine Forschungsergebnisse, sondern probiert eine - meist entsprechend vorläufig, konjunktivisch und in der ersten Person formulierte - These aus, in der Erwartung, dadurch kritische oder affirmative Resonanz zu erzeugen. Insoweit lässt sich ein Blogpost in gewisser Weise mit einer KonferenzPräsentation mit anschließender Diskussion vergleichen, allerdings mit einem unbegrenzten Diskutantenkreis und ohne die Notwendigkeit, sich und seine Mitdiskutant_innen zu einer bestimmten Zeit an einem bestimmten Ort zu versammeln.

Dabei bleibt, wie Ino Augsberg zurecht feststellt, das Bloggen trotzdem notwendig der Schriftlichkeit verhaftet. Ohne die Frage nach dem Verhältnis von Schrift und Rede als Ausdruck einer gleichsam dahinterstehenden Sprache $^{34}$ an dieser Stelle umfassend $\mathrm{zu}$ behandeln, lässt sich vielleicht festhalten: wenn man mit Derrida $^{35}$ der Schrift zuspricht, gleichzeitig Möglichkeit und Unmöglichkeit von Sprache und Semiosis überhaupt zu explizieren $^{36}$, dann gilt das trotz der Betonung des intersubjektiven, diskursiven Elements auch für den Blog. Denn der Gedanke verflüchtigt sich auf dem Blog eben nicht als gesprochener. Vielmehr birgt der Blog das Potenzial, vor einzelnen Gedanken innezuhalten und sie in einer wenngleich möglicherweise vorläufigen Form zum Ausdruck $\mathrm{zu}$ bringen. Der englische Juraprofessor und Blogger Mark Elliott sieht genau hierin den Mehrwert seines eigenen Blogs: der Blog ermögliche ihm, etwa in der Auseinandersetzung mit einzelnen Urteilen, Berichten oder ähnlichen Entwicklungen, sich Teile größerer Rechercheprojekte $\mathrm{zu}$ erarbeiten und Gedanken im Wege eines iterativen Schreibprozesses festzuhalten, möglicherweise

34 Krämer Medium, Bote, Übertragung - Kleine Metaphysik der Medialität (2008), 22.

35 Der Sprachphilosoph Jacques Derrida beschäftigte sich in seinem Werk »De la grammatologie« (1967) mit dem Verhältnis zwischen Schrift und Sprache. Er stellt die These auf, dass die abendländische Philosophie unter einem "Logozentrismus«, einer einseitigen Beschäftigung mit der gesprochenen Sprache, leide und deswegen die Schrift nur als ein Derivat der Sprache verstehe. Demgegenüber geht Derrida von der Schrift aus, indem er verschiedene kanonische Werke der abendländischen Philosophie, insbesondere die Werke JeanJacques Rousseaus, im Wege der durch Derrida selbst geprägten Methode der Dekonstruktion analysiert.

36 Krämer aaO, 24; Krämer Sprache, Sprechakt, Kommunikation (2001), 218. Augsberg bezieht sich explizit auf diese Derrida-Interpretation. 
als Vorstufe zu wissenschaftlichen Aufsätzen oder Buchprojekten ${ }^{37}$.

Womit wir bei einem der wohl wirkmächtigsten Argumente der Blogkritiker_innen angelangt sind: ist es denn dann Wissenschaft, was auf juristischen Blogs betrieben wird? Sind nicht Aufsätze, Monographien, mehrbändige Sammelwerke, Kommentare, kurz: das, was wir als Kanon wissenschaftlicher Publikationsformen kennen, die Medien, in denen sich die rechtswissenschaftliche Erkenntnis ihren Ausdruck verschafft? So betonte jüngst Johannes Masing, dass es sich bei einem Vermerk über das GoogleUrteil des Europäischen Gerichtshofs ${ }^{38}$ um »eine schnell geschriebene, erste vorläufige Einschätzung" handele, nicht aber um einen »endgültig durchgearbeitete[n] Text, der nach wissenschaftlichen Standards ausgeformt ist ${ }^{39}$. Für Augsberg verlässt der Blog spätestens dort den Bereich rechtswissenschaftlichen Austauschs, wo aufgrund der kurzen zeitlichen Distanz die Reaktion auf ein aktuelles Ereignis (oder einen aktuellen $\mathrm{Text}^{40}$ ) in einen polemischen Duktus umschlägt und dadurch mehr als politische Talkshow denn als seriöse wissenschaftliche Arbeit anmutet. Auch in der Auftaktveranstaltung zu dem Forschungsprojekt, dem die Autor_innen angehören, wurde eine ähnliche Kritik formuliert: allenfalls könne das Bloggen dazu dienen, wissenschaftliche Auseinandersetzungen anzustoßen, einen Vorgeschmack für die eigentliche, darauffolgende Wissenschaft zu bieten, nicht aber: selbst Wissenschaft zu sein ${ }^{41}$.

Dass es jeder Blogpost an gedanklicher Durchdringungstiefe mit einer Monographie aufnehmen kann, das

37 Elliott VerfBlog (2014) http://www.verfassungsblog.de/academiclawyers-blog/.

38 EuGH 2014, Rs. C-131/12 (Google Spain SL).

39 Masing VerfBlog (2014) http://www.verfassungsblog.de/ribverfgmasing-vorlaeufige-einschaetzung-der-google-entscheidung-deseugh/.

40 Als Beispiele mögen etwa die Repliken von Katharina Mangold auf einen Beitrag Christian Hillgrubers in der Frankfurter Allgemeinen Zeitung oder von Katharina Mangold und Nora Markard auf einen Beitrag Kay Hailbronners dienen: Mangold VerfBlog (2014) http:// www.verfassungsblog.de/verfolgte-unschuld-vom-lande-oder-wa rum-es-keines-grundrechts-auf-diskriminierung-bedarf/ und Mangold/Markard VerfBlog (2014) http://www.verfassungsblog.de/die-ret tung-des-schweizer-kaeses-durch-die-haerteklausel/.

41 Mitschnitt der Podiumsdiskussion auf www.verfassungsblog.de/ podium-rechtswissenschaft-und-medienwandel. Vgl. auch den Bericht zu der Veranstaltung von Tina Winter Juwissblog (2013) http:// www.juwiss.de/bloggen-eine-neue- form-rechts-wissenschaftlichins-gespraech-zu-kommen/. Ähnlich auch Jean D'Aspremont in Bezug auf völkerrechtliche Blogs, D’Aspremont EJIL:Talk! (2011) http://ejil talk.org/in-defense-of-the-hazardous-tool-of-legal-blogging/: »legal blogging should not be construed as an ersatz of legal scholarship...it is not proper legal scholarship«. behauptet wohl keiner der aktuellen rechtswissenschaftlichen Blogs. Allerdings sind die Rechtswissenschaften mehr als nur die Summe ihrer schriftlichen Endprodukte. Sie arbeiten in Institutionen, mit bestimmten Praktiken und in bestimmten Formen, wobei die Frage danach, was genau unter Rechtswissenschaften in Abgrenzung zur Rechtsanwendung zu verstehen ist und ob darüber hinaus ein Bedarf für ihre Theoretisierung überhaupt besteht, freilich unter ihren Protagonist_innen umstritten ist ${ }^{42}$. Man kann die Frage nach der Wissenschaftlichkeit einzelner Blogposts offen lassen, und trotzdem vermuten, dass Blogs Auswirkungen (aus unserer Sicht durchaus positive) auf die Rechtswissenschaften und ihre Verortung sowohl in Hinblick auf die Praxis als auch auf Nachbardisziplinen sowie die politische Öffentlichkeit haben. Inwieweit sich diese Auswirkungen beschreiben lassen, wird derzeit aus ethnographischer Sicht im Rahmen eines Forschungsprojekts, das am Verfassungsblog angesiedelt ist, beforscht ${ }^{43}$.

Als normative Wissenschaft, die gerade von Diskurs und Kontroverse lebt, können die Rechtswissenschaften von einer bereits im Medium Blog angelegten gegenseitigen Bezugnahme, der Möglichkeit der Kontrastierung verschiedener Positionen, profitieren.

Blogs bieten sich auch an, um sich als Gruppe im rechtswissenschaftlichen Diskurs zu behaupten und etablierte Grenzen zu überwinden. Das ist erkennbar die Intention hinter dem JuWiss-Blog: es geht hier darum, einer im öffentlichen Diskurs sonst allenfalls marginal wahrgenommenen Gruppe, dem wissenschaftlichen Nachwuchs, ein eigenes Forum zu bieten. Eine ähnliche Motivation lässt sich auch hinter dem Projekt IntLawGrrls beobachten, einem amerikanischen Blog, der ausschließlich Texte von Völkerrechtlerinnen veröffentlicht ${ }^{44}$.

Ein gewisser Trend zeichnet sich in Deutschland ab, was den redaktionellen Hintergrund der Blogpublikation betrifft. Anders als die Einzel- und Gruppenblogs von einst, auf denen jede_r Blogger_in für sich verantwortet, was er/sie schreibt, experimentieren Projekte wie JuWissBlog, Verfassungsblog oder Völkerrechtsblog mit redaktionellen Strukturen, betreiben aktiv Themen- und Autor_innenakquise, redigieren die Texte und nähern sich konzep-

42 Siehe hierzu Jestaedt/Lepsius (Hrsg.) Rechtswissenschaftstheorie (2008) und speziell für das Öffentliche Recht Funke/Lüdemann (Hrsg.) Öffentliches Recht und Wissenschaftstheorie (2009).

43 Verfassungsblog: Perspektiven der Wissenschaftskommunikation in der Rechtswissenschaft, http://www.verfassungsblog.de/for schungsprojekt-verfassungsblog-perspektiven-der-wissenschaftskom munikation-der-rechtswissenschaft/ueber-uns/.

44 http://ilg2.org. 
tionell journalistisch getriebenen Online-Magazinen an. Das gilt z.B. auch für den 2006 aus einem studentischen Projekt hervorgegangenen Informations- und Medienrechts-Blog Telemedicus ${ }^{45}$. Beim Völkerrechtsblog durchlaufen die Beiträge mit nur wenigen Ausnahmen gar ein peer review-Verfahren, d.h. die Texte werden vor ihrer Veröffentlichung auf dem Blog von Mitgliedern des eigens dazu eingerichteten Beirats redigiert, der hauptsächlich aus einer Reihe von Habilitierenden oder jungen Professor_innen besteht.

$45 \mathrm{http}: / /$ www.telemedicus.info.
Blogs tragen dazu bei, dass sich die Öffentlichkeit, in der Rechtswissenschaft stattfindet, verändert. Sie machen den Wissenschaftsdiskurs, in dem sich Rechtswissenschaftler_innen untereinander auf Problemstellungen, Lösungsansätze und Argumentationstopoi verständigen, zugänglich für das Informations- und Meinungsbildungsbedürfnis einer breiteren politischen Öffentlichkeit. Und umgekehrt verschaffen sie der Wissenschaft und ihrer Expertise einen enormen zusätzlichen Resonanzraum. Auch in dieser Hinsicht wirken Blogs somit als Mittel, Grenzen durchlässiger zu machen. 